Original Contribution

\title{
THE EFFECT OF A SOLUTION-FOCUSED APPROACH TO THE DYNAMICS IN THE SEVERITY OF ANXIETY AND DEPRESSION SYMPTOMS AT ALCOHOL DEPENDENCE
}

\author{
V. Dineva* \\ Pedagogy, Psychology and History Department, „Angel Kanchev” University of Ruse, Bulgaria \\ Mental Health Center, Ruse, Bulgaria
}

\begin{abstract}
Purpose: To examine the effect of the Solution-Focused Approach to the dynamics in the severity of anxiety and depression symptoms in people with alcohol dependence.

Methods: The research was carried out with 60 voluntarily hospitalized patients with alcohol dependence. Before their admission to the Ward Addiction Treatment, a HADS scale recorded the presence of anxiety and depression symptoms. Patients were included on random in experimental and control groups with stratification of identity counting the demographic factors and the characteristics of their illness. Medication therapy was applied to both groups in compliance with their personal needs. On the $7^{\text {th }}$ and $14^{\text {th }}$ day of the treatment, the experimental group was additionally involved in two one-hour solution-focused individual sessions without other psychological interventions. On the $1^{\text {st }}, 10^{\text {th }}$ and $20^{\text {th }}$ day of the treatment, the HAM-A scale was applied to assess the severity of the anxiety symptoms, as well as the HAM-D 17 scale for depression symptoms. Variations at $\mathrm{p} \leq 0.01$ were considered statistically significant.

Results: The severity of the anxiety and depression symptoms was reduced in the experimental group thanks to the effect of the solution-focused sessions.

Conclusions: On a pharmacotherapy background, the solution-focused approach to alcohol dependence lessens the severity of anxiety and depression symptoms more effectively than the solely medication intake.
\end{abstract}

Key words: Solution-Focused Approach, alcohol dependence, anxiety, depression

\section{INTRODUCTION}

The relationship of alcohol dependence with depression and anxiety conditions is frequently discussed in the psychological and medical literature. Conditions related to the use of psychoactive substances (PAS), anxiety (1-4) and depression (5) disorders are particularly comorbid for psychiatric patients and general populations as well. According to data from a large-scale epidemiological study conducted in the United States on alcoholism and related disorders, the existence of PAS dependence increases three times the risk of a simultaneously developed anxiety disorder (2). Severe depression occurs two to four times more often

*Correspondence to: Vanya Dineva, 8 Studentska str., ,Angel Kanchev” University of Ruse, Office 1.203.1, Ruse 7017, Bulgaria, Tel +359 889 266 832,e-mail:vdineva@uni-ruse.bg among people with alcohol or other dependence than amongst the general population (5), affecting $30-45 \%$ of the people who seek treatment for PAS dependence (2). The presence of a depression and/or anxiety disorder increases the risks of relapse in alcohol dependence (6). Besides, patients need not only pharmacotherapy but also the adoption of adequate strategies for resolving associated problems considering that their inability to cope with daily stress and real life difficult situations can be a factor for developing comorbid disorders, especially when some of them take alcohol trying to manage the stress and calm down the occurring condition (7). In this context, the application of psychotherapeutic interventions supplementary to the medication therapy can reduce the anxiety and depression symptoms, as well as help patients better understand the relation 
between psychiatric symptoms and the alcohol addiction so that they can achieve a long-term remission, respectively, improve the quality of their life (8).

Amongst the variety of psychotherapeutic modalities applied in practice regarding alcohol dependence, the Solution-Focused Approach (SFA) is distinguished by its pragmatism. Created in the $80 \mathrm{~s}$ of $20^{\text {th }}$ century by Steve de Shazer, Insoo Kim Berg and their colleagues from the Brief Family Therapy Center in Milwaukee, the approach develops the idea that it is not necessary to know what the problem in substance is in order to find its solution for a specific live situation. Minding that every man's reality is created in their thoughts, the more people talk about solutions, the more ideas are generated to the extent of a self-belief that they can manage to realize them in practice. The main principle of the method is to involve individual goals, resources, skills, abilities, strengths, and successes for coping with the problem. This is why the therapeutic process focuses on "conversations about solutions" by visualizing client's desired future and "conversations about changes", which allow finding solutions as such (9). It is the key for working in every unique situation, so as to help alcohol dependent people find and adapt in practice those realistic strategies, which are relevant to their specific needs for improving the quality of their life.

The goal of this research is to examine the effect of the Solution-Focused Approach to the dynamics in the severity of anxiety and depression symptoms in patients with alcohol dependence.

\section{MATERIALS AND METHODS}

Sixty voluntarily hospitalized patients with the syndrome of alcohol dependence were examined after a primary test for the presence of anxiety and depression symptoms before their admission to the Ward Addiction Treatment. According to their personal needs, specific medication therapy was prescribed to all of them and it was applied under the supervision of the medical personnel. People were included on random in two groups - an experimental group (31 persons) and a control group $(29$ persons $)$ with stratification of identity counting the demographic factors and the characteristics of their illness. All persons had intellectual quotient within the frames of the average norm and they were not registered for cognitive disorders. During a period of 20 days supplementary to the pharmacotherapy, each person from the experimental group was involved in two one-hour individual solutionfocused sessions (on $7^{\text {th }}$ and $14^{\text {th }}$ day) without other psychological interventions. No sessions were conducted with the control group members who were treated only with relevant medications according to their specific needs.

The following instruments were used in the research settings:

1. Hospital Anxiety and Depression Scale (HADS) - a self-assess questionnaire for identifying severe anxiety and depression states designed by A. S. Zigmond and R. P. Snaith in 1983 (10). Patients were instructed to spontaneously choose one answer from the four suggestions proposed for each of the fourteen questions. The assessment was made in conformity with the Anxiety and Depression Scale after summing up the scores corresponding to the chosen answer. The questionnaire was used once - in the beginning of patient admission for treatment;

2. Hamilton Anxiety Rating Scale (HAM-A), a measure assuring a quantitative assessment for the severity of the anxiety symptoms. It is a widely applied and acknowledged method in the clinical practice for patient state tracking. Patients are assessed according to 14 items of anxiety symptoms, and after summing up the scores to each item, the total result serves for evaluation of therapeutic response (11);

3. Hamilton Depression Rating Scale, 17 item (HAM-D 17), a measure assuring a quantitative assessment for tracking the dynamics of patients' state before the therapeutic interventions, in the process of their performance and after them regarding patients' mood, feeling of guiltiness, suicidality, insomnia/hypersomnia, agitation, activity, psychosomatics, and other indices (12).

HAM-A and HAM-D 17 scales were applied on $1^{\text {st }}, 10^{\text {th }}$ ad $20^{\text {th }}$ day of the treatment at the Ward Addiction Treatment. The data obtained was processed by relevant methods of mathematics and statistics using SPSS Statistics 23.0. Variations are considered statistically significant at $\mathrm{p} \leq 0.01$. Frequency and non-parametric analysis was applied, while the relation strength was measured through coefficients of correlative type enabling to ascertain if it is regular and not of random type. 


\section{RESULTS AND DISCUSSION}

The anamnestic data for the patients from the experimental group (EG) and from the control group $(\mathrm{CG})$, gathered at their admission to the Ward Addiction Treatment, include characteristics such as gender, age, education, marital status, employment, number of hospitalizations for detoxification regarding alcohol dependence syndrome, as well as level of anxiety/depression indices (acc.to HADS questionnaire). In summary, the results can be listed as it follows:

- for both groups the prevailing percentage is of male representatives: $80.6 \%$ from the EG and $82.8 \%$ from the $\mathrm{CG}$;

- the age index shows that the most frequent result concerns patients of 25 - 35 years (35.5\% from the EG and $34.5 \%$ from the CG). The second place is for representatives within 46 - 55 years $(32.3 \%$ from the EG and $31.0 \%$ from the $\mathrm{CG}$ ) followed by representatives within 36 - 45 years (EG - 25.8\% and CG $27.6 \%$ ). The most insignificant percentage concerns people within 56 - 65 years of age (EG - 6.5\% and CG - 8.9\%);

- most of the patients have secondary education (54.8\% from the EG and 55.2\% from the CG), followed by the close percentage of persons with elementary education $(22.6 \%$ from the EG and $20.7 \%$ from the $\mathrm{CG}$ ) and higher education (EG $22.6 \%$ and $\mathrm{CG}-24.1 \%$ );

- the percentage of married people is the highest $(41.9 \%$ from the EG and $37.9 \%$ from the CG), followed by the close percentage of divorced and single people $(29.0 \%$ from the EG and $31.0 \%$ from the CG);

- employed people appear as the most frequent result $-51.6 \%$ from the EG and $51.7 \%$ from the CG;

- the highest percentage of examined persons is for patients with a second successive hospitalization for detoxification (35.5\% from the EG and $37.9 \%$ from the CG), followed by patients with a first hospitalization $(22.6 \%$ from the EG and $20.7 \%$ from the CG) and two previous detoxification (19.4\% from the EG and $17.2 \%$ from the CG). The most insignificant share is for patients with a fourth successive hospitalization $-9.7 \%$ from the EG and $10.3 \%$ from the CG;

- most of the people admitted for treatment express signs of a mild severity of anxiety (41.9\% from the EG and $44.8 \%$ from the CG) and depression (48.4\% from the EG and $51.7 \%$ from the CG). Moderate severity is shown by an identical percentage of people with anxiety, and slightly smaller percentage of people with depression (35.5\% from the EG and 37.9\% from the CG). The lowest percentage is for people with severe indices.

After statistical data processing and application of non-parametrical analysis, the results show availability of a statistically significant relation (at $\mathrm{p} \leq 0.01$ ) for an existing identity of the tested people profile that gives reasons for carrying out the research as intended.

Besides the implemented medication therapy prescribed according to patients' personal needs, HAM-A and HAM-D 17 scales were applied to both groups on $1^{\text {st }}, 10^{\text {th }}$ and $20^{\text {th }}$ day of their hospitalization to measure the severity of their anxiety and depression state. Additional interventions were not made to the patients from the control group, but two onehour solution-focused individual sessions were conducted with the people from the experimental group. In general, the sessions aimed to:

- understand what expectations and potential benefits from the treatment the interviewed patients might have;

- visualize their desired future when the current existential problem has already been coped with;

- define the goals as well as the time distance between them and the present state by finding out the difference and noticing the change in what has seemed a monotony.

- discuss the moments when the problem was not so serious (even missing) as examples of desired targets achieved in the past;

- focus on things which have already been made placing an emphasis on the achievements and personal responsibility. Part of SFA mastery is to discover patients' moments of success

- at a subsequent collapse/relapse to lead the conversation so that to identify in details the similarities and differences with previous key locations, events, persons, quantity of alcohol consumed, etc. Collapses/relapses are discussed not like a failure but an emerging opportunity for a new better beginning motivated by a gained experience;

- include compliments related with what has been achieved to the moment in the attempts to resolve a problematic situation, make an acknowledgement for its complexity and suggest further specific behavioral activities. 
The data shows that in the very beginning the signs of a mild severity is identified for the bigger part of the examined people with anxiety $(48.4 \%$ from the EG and $51.7 \%$ from the CG) and depression (48.4\% from the EG and $48.3 \%$ from the CG), followed by patients with moderate levels of anxiety and depression. The portion of people with severe indices is the smallest.
In comparison with the $1^{\text {st }}$ day of SFA inclusion, during the next tracking procedures the recorded progress level in the dynamics of anxiety and depression indices is different for the two groups. On $10^{\text {th }}$ day of hospitalization, the moderate severity of anxiety symptoms in the experimental group is 1.18 times lower and on $20^{\text {th }}$ day -1.66 times. In the control group, the indices are respectively 1.13 times lower at the second measurement, and 1.35 times - at the third one (Figure 1).

HAM-A PROFILE

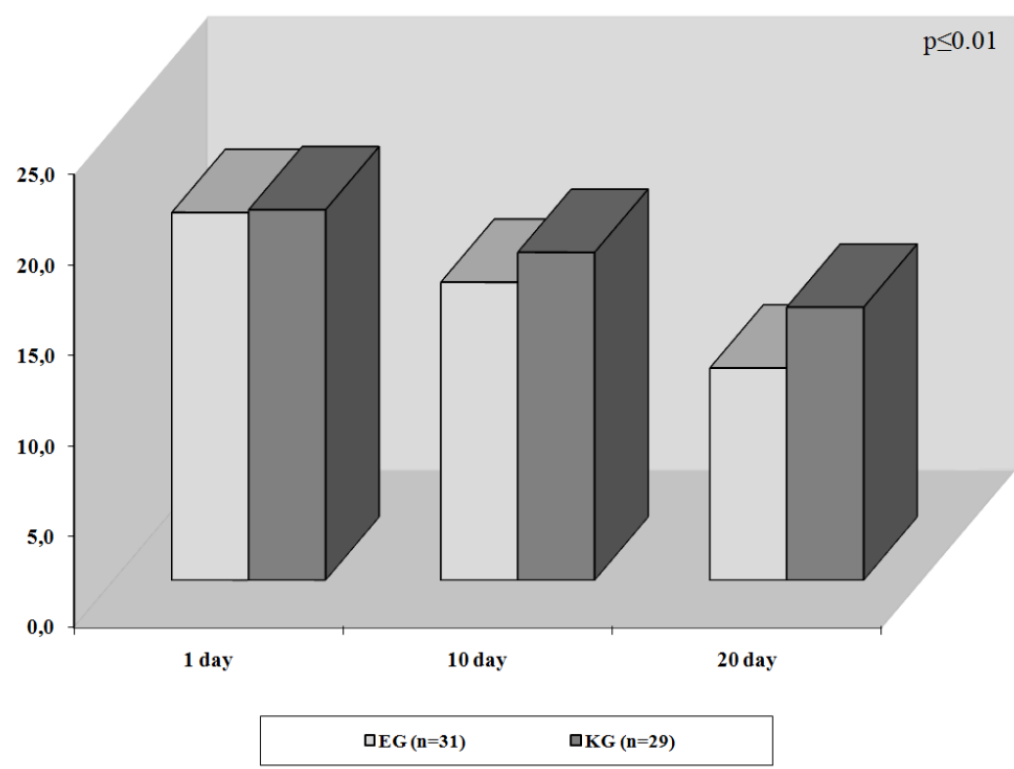

Figure 1. Dynamics in anxiety indices acc. to Hamilton Anxiety Rating Scale (HAM-A)

The ratio is similar for the averaged summed-up results of the depression severity indices. They are 1.3 times lower for the EG on $10^{\text {th }}$ day and 1.97 times - on $20^{\text {th }}$ day. The indices in the CG are respectively 1.19 times lower at the second measurement and 1.4 times - at the third one (Figure 2).

HAM-D PROFILE

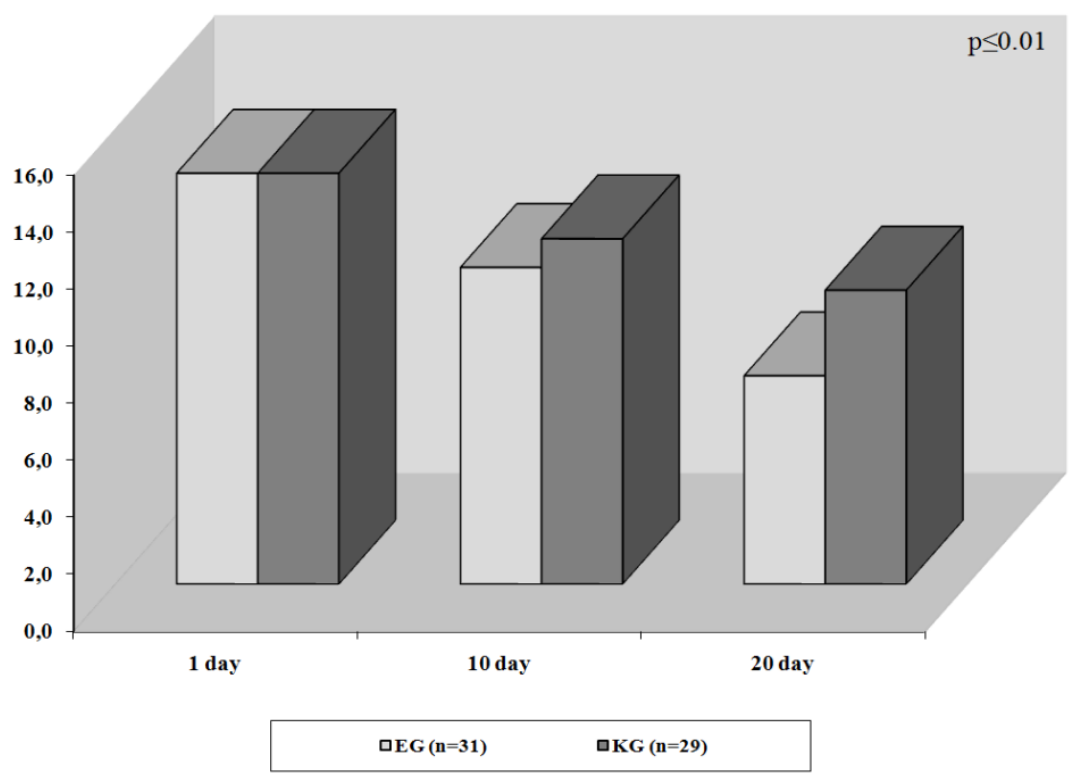

Figure 2. Dynamics in depression indices acc.to Hamilton Depression Rating Scale (HAM-D 17) 
After processing the data from the investigation, a statistically significant relation $(\mathrm{p} \leq 0.01)$ is estimated between:

- the age, number of successive hospitalizations and the anxiety and depression indices acc. to HAM-A and HAM-D scales. The strength of relation is indicated by the indices' values of correlative type, which vary within $0.571-0.718$ giving grounds for its interpretation as being substantial. It urges the necessity of applying appropriate therapeutic interventions (pharmacological and/or non-pharmacological) during the period of remission, no matter whether the anxiety and depression symptoms are determined by alcohol consumption or they become a reason for them. Thus, it is possible to influence the chronic-relapsing development of the disorder so as to achieve a long-term abstinence, respectively improvement of the addict's quality of life.

- the initially and subsequently measured $\left(10^{\text {th }}\right.$ and $20^{\text {th }}$ day) indices of anxiety and depression symptoms acc. to HAM-A and HAM-D scales. The indices' values vary within $0.712-0.806$ and the relation strength is interpreted as being high. The follow-up measurements record a descent in the severity of the anxiety and depression symptoms that appears more effective for the patients in the experimental group (EG). These results reason the assumption that the improvements of the patients in the EG are owed not only to the medication therapy but the effect of the two solution-focused individual interventions.

\section{CONCLUSION}

In summary, it can be generalized that on the background of pharmacotherapy the SolutionFocused Approach to patients with alcohol dependence is more efficient in reducing the severity of the anxiety and depression symptoms than the solely intake of medications. Its application is based on a constructed framework with a focus on the discourse that helps patients commit themselves with a high rate of reflexivity to make progress in their life functionality. Focusing the conversation on matters of personal significance and motivating patients to find a solution appropriate to their necessities, effect what they believe or do. In this context, aiming to achieve time-sustainable results for people with chronic state such as alcohol dependence, it is better when the application of the SolutionFocused Approach includes a sequence of therapeutic cycles related to cognition, maintenance and support of new behaviors for improving patients' psychosocial functionality, respectively, their quality of life.

\section{REFERENCES}

1. Fatseas, M., Denis, C., Lavie, E. and Auriacombe, M., Relationship between anxiety disorders and opiate dependence-a systematic review of the literature: implications for diagnosis and treatment. $J$ Subst Abuse Treat, 38(3):220-230, 2010.

2. Grant, B.F., Stinson, F.S., Dawson, D.A., Chou, S.P., Dufour, M.C., Compton, W. Pickering, R.P. and Kaplan, K., Prevalence and co-occurrence of substance use disorders and independent mood and anxiety disorders: results from the National Epidemiologic Survey on Alcohol and Related Conditions. Arch Gen Psychiatry, 61(8):807-816, 2004.

3. Marmorstein, N.R. Anxiety disorders and substance use disorders: different associations by anxiety disorder. $J$ Anxiety Disord, 26(1):88-94, 2012.

4. Martins, S.S. and Gorelick, D.A. Conditional substance abuse and dependence by diagnosis of mood or anxiety disorder or schizophrenia in the U.S. population. Drug Alcohol Depend, 119(1-2):28-36, 2011.

5. Compton, W.M., Thomas, Y.F., Stinson F.S. and Grant, B.F., Prevalence, correlates, disability, and comorbidity of DSM-IV drug abuse and dependence in the United States: results from the national epidemiologic survey on alcohol and related conditions. Arch Gen Psychiatry, 64(5):566-576, 2007.

6. Kushner, M.G., Abrams, K., Thuras, P., Hanson, K.L., Brekke, M. and Sietten, S., Follow-up study of anxiety disorder and alcohol dependence in comorbid alcoholism treatment patients. Alcohol Clin Exp Res, 29(8):1432-1443, 2005.

7. DeMartini, K.S. and Carey, K.B., The role of anxiety sensitivity and drinking motives in predicting alcohol use: a critical review. Clin Psychol Rev, 31(1):169-177, 2011.

8. Hesse, M. Integrated psychological treatment for substance use and co-morbid anxiety or depression vs. treatment for substance use alone. A systematic review of the published literature. BMC Psychiatry, 9:6, 2009.

9. Corey, G., Theory and Practice of Counseling and Psychotherapy. CENGAGE Learning, Mason, OH, United States, 2018.

10.Zigmond, A.S. and Snaith, R.P. The hospital anxiety and depression scale. Acta Psychiatr Scand, 67(6):361-370, 1983

11.Hamilton, M. The assessment of anxiety states by rating. $\mathrm{Br} \mathrm{J}$ Med Psychol, 32(1):50$55,1959$.

12. Hamilton, M. A rating scale for depression. $J$ Neurol Neurosurg Psychiatry, 23:56-62, 1960. 